\title{
Aplicación de algoritmos paramétricos en el diseño estructural. La Ópera de Sídney desde una perspectiva contemporánea
}

\section{Application of parametric algorithms in structural design. The Sydney Opera House from a contemporary perspective}

\author{
$\underline{\text { J. Rey-Rey }}^{(*)}$ \\ RESUMEN
}

La Ópera de Sídney puede considerarse un paradigma en relación a la influencia que los métodos de representación gráfica y análisis estructural ejercen en la concepción y construcción de las obras de arquitectura. Se plantean posibles esquemas estructurales para las cubiertas de dicho edificio respetando la forma propuesta originalmente por Utzon, forma que se vio modificada durante el proceso de proyecto para hacer viable su construcción, poniendo en evidencia el impacto que los avances científicos y tecnológicos acaecidos durante la segunda mitad del siglo pasado han tenido en la manera de concebir y construir edificios.

Palabras clave: Ópera de Sídney; diseño paramétrico; análisis estructural; láminas de hormigón.

\section{ABSTRACT}

The Sydney Opera House can be considered a paradigm for the influence methods of drawing and structural analysis have on architectural design and construction. This paper presents various structural scheme designs for the roof of the Sydney Opera House based on the original geometry proposed by Utzon. This geometry was modified in the design process in order to make its construction viable, demonstrating the impact that the scientific and technological progress of the second half of the 2oth century have had in the design and construction of buildings.

Keywords: Sydney Opera House; parametric design; structural analysis; concrete shells.

${ }^{(*)}$ Escuela Técnica Superior de Arquitectura - Universidad Politécnica de Madrid (España). Persona de contacto/Corresponding author: juan.rey@upm.es (J. Rey-Rey)

Cómo citar este artículo/Citation: Rey-Rey, J. (2014). Aplicación de algoritmos paramétricos en el diseño estructural. La Ópera de Sídney desde una perspectiva contemporánea. Informes de la Construcción, 66(EXTRA-1): mo16, doi: http://dx.doi.org/10.3989/ ic.13.093.

Licencia / License: Salvo indicación contraria, todos los contenidos de la edición electrónica de Informes de la Construcción se distribuyen bajo una licencia de uso y distribución Creative Commons Reconocimiento no Comercial 3.o. España (cc-by-nc). 


\section{INTRODUCCIÓN}

El importante desarrollo tecnológico e industrial surgido especialmente durante la segunda mitad del siglo pasado ha eliminado las históricas limitaciones técnicas en el ámbito de los proyectos arquitectónicos, desembocando en la situación actual en la que cualquier planteamiento formal puede ser analizado desde un punto de vista estructural, concluyéndose por tanto que ha desaparecido la barrera del análisis en lo que al desarrollo de un proyecto arquitectónico se refiere (1).

En la actualidad, al igual que a finales del siglo XIX, nos encontramos en un periodo de transición, y también, como entonces, es la tecnología la que orienta el cambio. No la tecnología de los nuevos materiales (hormigón y acero) como sucedía tras la revolución industrial sino que es la nueva tecnología digital aplicada a los sistemas de diseño, cálculo y fabricación la que está siendo el motor de la actual transformación. Hoy no es tanto el paradigma mecanicista el que prevalece en muchos casos en la concepción de los edificios (2) sino que nuevos elementos como la tecnología digital integrada está cambiando la forma de diseñar y concebir el entorno construido (3).

Ante este contexto cabría plantearse las siguientes cuestiones: ¿Puede el diseño paramétrico y la tecnología CAD-CAM-CAE en conjunción con los programas actuales de análisis estructural basados en los Métodos Matricial y de los Elementos Finitos hacer más sencilla la construcción de estructuras ligeras y eficientes hoy en día? ¿Puede la tecnología digital ayudar a ampliar el abanico formal a la hora de diseñar edificios y a la vez permitir el uso de sistemas estructurales racionales que optimicen el consumo de materiales bajo dichas premisas? (4).

\section{LA ÓPERA DE SÍDNEY}

La Ópera de Sídney, obra del arquitecto danés Jørn Utzon, puede considerarse un paradigma en relación a la influencia que los métodos de representación gráfica y análisis estructural ejercen en la concepción y construcción de las obras de arquitectura (5). El edificio fue gestado en una época (19561973) inmediatamente anterior al desarrollo científico y tecnológico anteriormente referido $\mathrm{y}$, a pesar de emplearse de forma pionera ordenadores durante su desarrollo, tanto el software como el hardware no estaban lo suficientemente maduros. Así, el proyecto del edificio debió elaborarse con medios básicamente manuales adoptando en consecuencia importantes simplificaciones, tanto en lo referente a la representación gráfica como al análisis estructural (6). Esta ausencia de herramientas de diseño disponibles acordes a la complejidad formal de la propuesta planteada condicionó enormemente la marcha del proyecto, dilatándose dramáticamente en el tiempo (hasta un total de 17 años) y multiplicándose su costo final de forma desproporcionada (en aproximadamente un $1.300 \%$ ) (7).

Además, la solución estructural finalmente construida dista mucho de la imaginada por Utzon inicialmente debido a las importantes modificaciones que debieron introducirse para hacer viable su análisis y representación (8). Así, donde Utzon imaginó una fina lámina de hormigón flotando sobre el paisaje se construyó finalmente una estructura más pesada, formada por costillas pretensadas de hormigón con unas secciones notablemente mayores (9). La geometría también debió ser modificada de forma ostensible, pasando de un trazado «libre» a una geometría esférica, tal y como se observa en la Figura 1.

Si este edificio se pretendiese construir en la actualidad, con total seguridad el curso de los acontecimientos se desarrollaría por senderos muy diferentes. Ante este supuesto se plantean posibles esquemas estructurales para las cubiertas de dicho edificio respetando la forma «libre» propuesta originalmente por Utzon así como su carácter laminar, poniendo en evidencia el impacto que los ordenadores personales y los programas informáticos asociados han tenido en la manera de concebir y construir edificios.

\section{ALGORITMOS DE DISEÑO PARAMÉTRICO}

El modelado de «formas libres» a través de superficies paramétricas permite una rápida generación de formas complejas con una mínima cantidad de datos. Así, los sistemas CADCAM-CAE han redefinido la concepción, representación, fabricación y construcción vinculadas al proyecto arquitectónico, haciendo accesible la manipulación de «formas libres» (10), incluso con conocimientos mínimos de geometría (con el riesgo implícito que este hecho conlleva).

El importante avance de la informática en las últimas décadas ha posibilitado a los arquitectos trabajar de forma relativamente sencilla e intuitiva con representaciones matemáticas de geometrías tridimensionales como las NURBS (Non-Uniform Rational B-Splines). Estas curvas y superficies orgánicas definidas por curvas vectoriales han permitido abordar

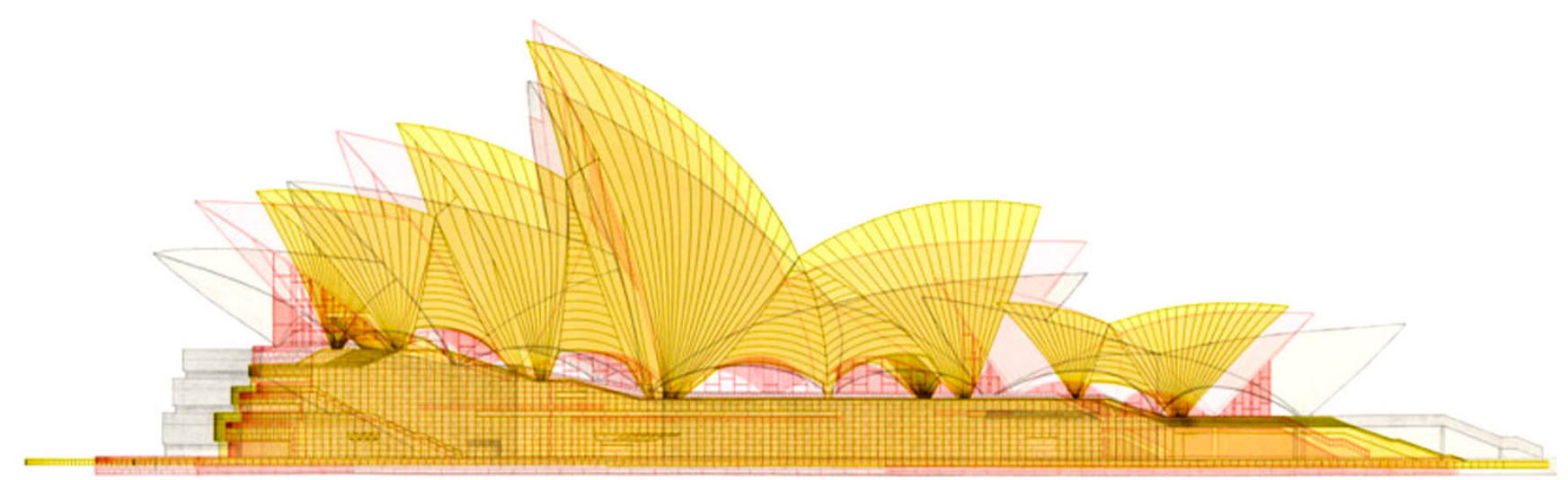

Figura 1. Comparativa de la evolución de la forma de las cubiertas de la Ópera de Sídney: concurso - en gris - (1956), geometría parabólica en rojo - (Libro Rojo, 1958) y geometría esférica -en amarillo- (Libro Amarillo, 1962). 
gráficamente la manipulación de espacialidades complejas, incluso desconociendo la especificidad analítica de sus ecuaciones paramétricas.

Pero, al contrario de lo que ha sucedido en otras industrias como la aeronáutica o la del automóvil, la construcción de edificios ha mostrado una inercia muy fuerte a integrar estos avances tecnológicos, particularmente durante las últimas décadas, dando continuidad al empleo de técnicas en algunos casos seculares. Indudablemente, este posicionamiento evita riesgos pero a su vez obstaculiza la incorporación de innovaciones y mejoras en los procesos constructivos.

El diseño paramétrico es por tanto una técnica para el control y la manipulación de objetos de diseño a cualquier escala, que emplea algoritmos como métodos de generación de formas y posibilita la producción de formas complejas basadas en reglas sencillas para el control de cada uno de sus componentes. Al definir una determinada forma mediante NURBS se obtiene un modelo geométrico dinámico, esto es: una forma definida por un conjunto de parámetros asociados, flexibles y modificables.

Como siempre que una innovación entra en escena, la inexistencia de referencias y precedentes genera tentativas fallidas y tratamientos superficiales de dicha nueva herramienta. El caso del diseño paramétrico en el ámbito arquitectónico no ha escapado de este cliché. Así, el diseño paramétrico ha sido empleado frecuentemente en ejercicios formales de dudoso interés, sin mayores pretensiones que la pura experimentación estética (11).

Incluso en fechas más recientes se ha extendido la tendencia de emplear las nuevas herramientas de diseño algorítmico para producir formas de gran complejidad partiendo de la implementación de reglas y fórmulas sencillas. Este enfoque ha conducido frecuentemente a diseños con una complejidad innecesaria, en los que ésta era además introducida sin un fundamento o motivación claros.

Existe por tanto la tentación de emplear la potencialidad de las nuevas herramientas de diseño paramétrico de una forma sencilla para aumentar artificialmente la complejidad de un diseño en lugar de hacer uso de éstas de una manera más intensa (y compleja) en aras de alcanzar diseños más simples, coherentes y en último término, eficaces.

\section{APLICACIÓN DE ALGORITMOS DE DISEÑO PARAMÉTRICO EN EL DESARROLLO DE PROPUESTAS PARA LA ESTRUCTURA DE LAS CUBIERTAS DE LA ÓPERA DE SÍDNEY}

\subsection{Introducción}

Aplicando estas técnicas es posible realizar exploraciones geométricas sobre superficies complejas en la búsqueda de soluciones eficaces (12). En el caso de las superficies propuestas por Utzon originalmente para las cubiertas de la Ópera de Sídney, dado que éstas no estaban definidas matemáticamente, el empleo de NURBS y geometrías parametrizadas sobre dicha superficie se adapta a la perfección a la problemática planteada.

Estas técnicas cobran todavía más relevancia en cuanto a la fabricación y construcción de la obra. Así, mientras que en la obra de construcción de las cubiertas de hormigón que hoy se pueden admirar en Bennelong Point, en el Sídney de los años sesenta, el proceso constructivo requirió la prefabricación de sus componentes para racionalizar y en último término hacer viable su construcción, los inicios de este nuevo siglo se caracterizan por poner el acento en los sistemas de fabricación digitales.

Dichos sistemas se caracterizan por centralizar toda la información relevante del edificio en un archivo de datos tridimensional, que además de para su análisis estructural, sirve también para alimentar directamente las máquinas de corte, doblado, etc. En cualquier caso es importante destacar que la no estandarización de piezas puede aumentar la complejidad del proceso de montaje si éste no está bien concebido.

Hasta hace no mucho tiempo, los medios de representación y análisis coincidían aproximadamente con las técnicas de construcción de su momento. En cambio, a día de hoy, la realidad es que las nuevas técnicas de representación y análisis estructural nos han llevado a un punto de desarrollo tal que la limitación que hasta ahora existía en estos campos en el desarrollo de los proyectos arquitectónicos ha desaparecido. Y hoy es la industria la que supone el lastre mayor para la construcción de edificios con «formas libres» como las propuestas por Utzon en su momento.

\subsection{Generación de un modelo digital de la geometría original de las cubiertas}

Se ha empleado la documentación gráfica que Jørn Utzon presentó al concurso de arquitectura convocado para la generación de un modelo digital de una parte de las cubiertas; en concreto las dos piezas de mayor tamaño, que se corresponden con la zona de acceso y escenario del auditorio principal. Se considera que los resultados obtenidos serán extrapolables al resto de piezas. En concreto, se han tomado los dibujos de plantas y alzados (13) y se han digitalizado empleando para ello el programa informático Rhinoceros v5.o (NURBS modelling for $M A C$ ).

El proceso de digitalización ha consistido en la captura de puntos directamente sobre los dibujos anteriormente indicados, una vez escalados estos previamente. Posteriormente, se han interpolado curvas partiendo de los puntos previamente capturados, tanto de los dibujos de plantas como de alzados.

Los resultados obtenidos, tal y como se observa en la Figura 2, se consideran suficientemente aproximados:

A continuación, se han generado superficies a partir de las curvas anteriores, proyectando dichas curvas en las tres direcciones principales del espacio. Se han obtenido las curvas espaciales resultantes de la intersección de las superficies extruidas anteriormente indicadas. Estas curvas constituyen las curvas maestras (aristas) para la definición de las superficies que constituyen las dos piezas de cubierta seleccionadas (ver Figura 3).

A partir de este conjunto de curvas en el espacio se han generado los fragmentos de superficie de cubierta enmarcados por dichas curvas. Para ello se ha empleado la opción de generación de superficies a partir de curvas maestras de Rhinoceros. Esta interpolación se considera válida por varios motivos: por no existir información suficiente para generarla de forma 

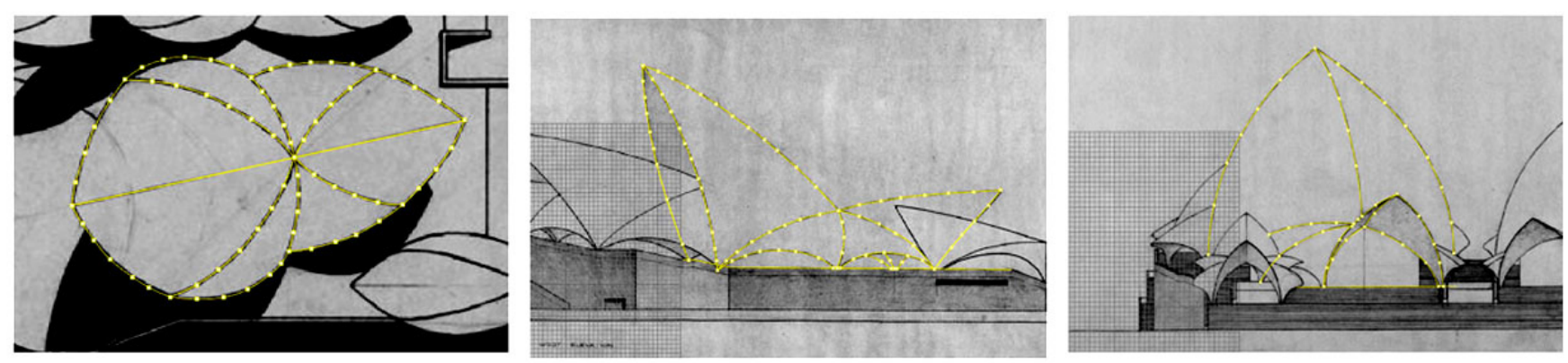

Figura 2. Proceso de digitalización de los dibujos originales.
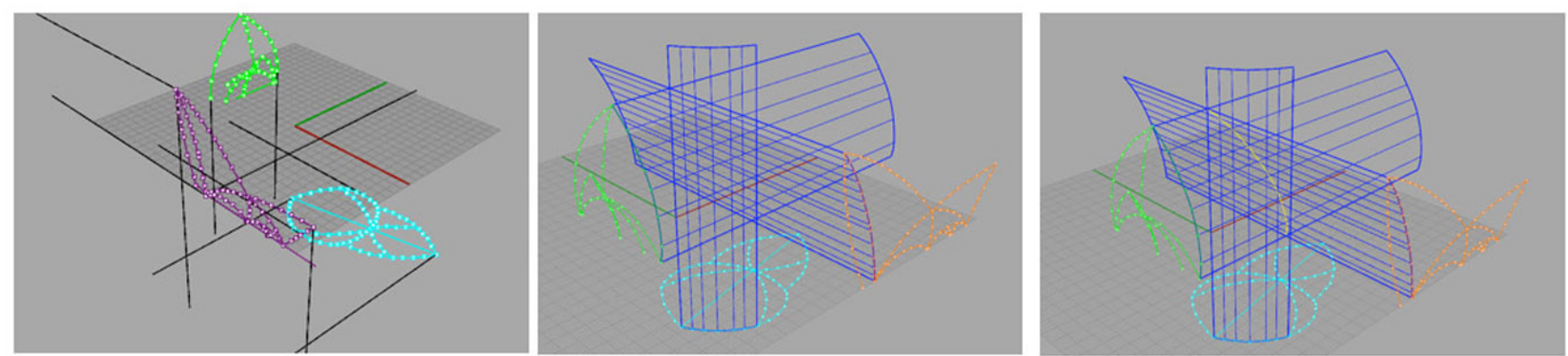

Figura 3. Montaje tridimensional de las tres proyecciones digitalizadas, extrusión de superficies y obtención de curvas de las aristas por intersección de dichas superficies.

precisa (por lo que la aquí presentada es una de las infinitas soluciones posibles al problema) y por tener un parecido razonable con las imágenes existentes.

Sobre estas superficies generadas se ha realizado un mallado en elementos finitos triangulares de tres nodos, para facilitar el posterior análisis estructural (14) (ver Figura 4).

\subsection{La estructura de cubierta de la Ópera de Sídney desde una perspectiva contemporánea}

Las técnicas de construcción de cubiertas ligeras en hormigón armado (láminas) se han basado tradicionalmente en el uso intensivo de mano de obra y consecuentemente se han visto abocadas a un paulatino abandono debido a su creciente coste. Según estudios realizados por Williamson en 2003 (15), el valor de la mano de obra (no reajustado por la inflación) aumentó entre 1958 y 2002 entre 8 veces (mano de obra no especializada) y once veces (mano de obra especializada), mientras que el coste de los materiales de construcción en el mismo periodo aumentó solamente entre 3,8 veces (acero) y 4,8 veces (hormigón).

Así, para que la construcción de cubiertas laminares de hormigón fuese viable hoy en día sería necesario crear técnicas de construcción menos demandantes de mano de obra, ya que la ejecución de cimbras y encofrados suponen porcentualmente una parte muy importante del coste de la estructura completa (16). Debido fundamentalmente a este motivo el autor entiende que hoy en día el proyecto de la estructura de las cubiertas de la Ópera de Sídney debería plantearse en acero.

Un aspecto importante a resaltar en relación a las cubiertas proyectadas por Utzon para la Ópera de Sídney es que, a diferencia de las cubiertas laminares de Nervi, Candela, Torroja, etc. (17), en las cubiertas de la ópera el hormigón armado estaba en principio previsto que no fuese visto: se preveía su recubrimiento exterior mediante un acabado cerámico y su interior iba a quedar oculto en su mayor parte por los imprescindibles falsos techos derivados de las necesidades de acondicionamiento acústico de las salas. En las áreas en las que dichos falsos techos no eran necesarios, Utzon, tal y como deja plasmado en sus dibujos de concurso, prevé tratar el interior de hormigón con un acabado de color dorado.

Así, hoy como entonces, parece más natural plantear una estructura metálica para generar una superficie del tamaño y forma propuestos por Utzon. Las ventajas fundamentales que presenta frente a la versión construida son:
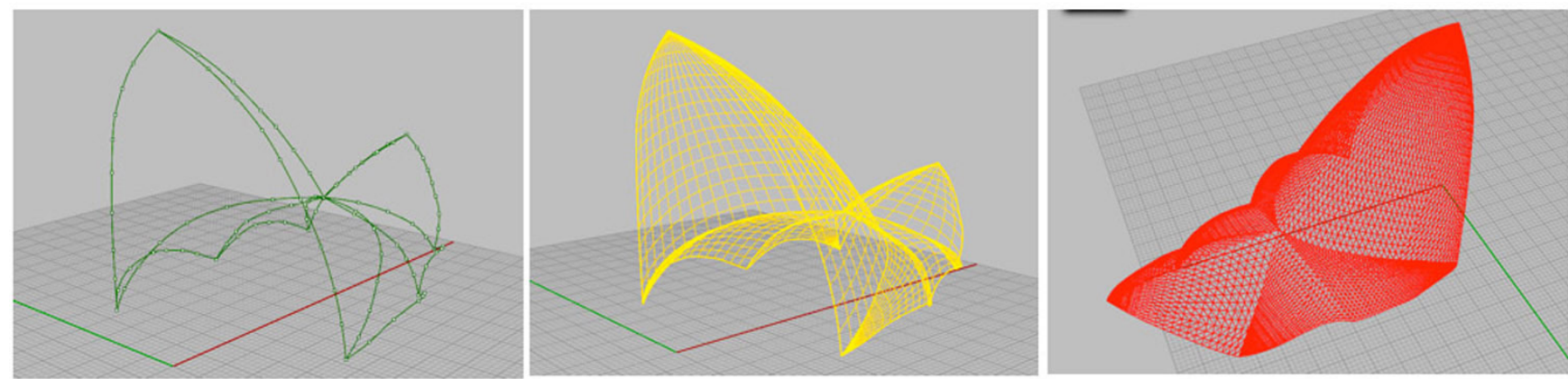

Figura 4. Curvas de las aristas, generación de las distintas porciones de superficie y mallado con elementos triangulares de tres nodos. 

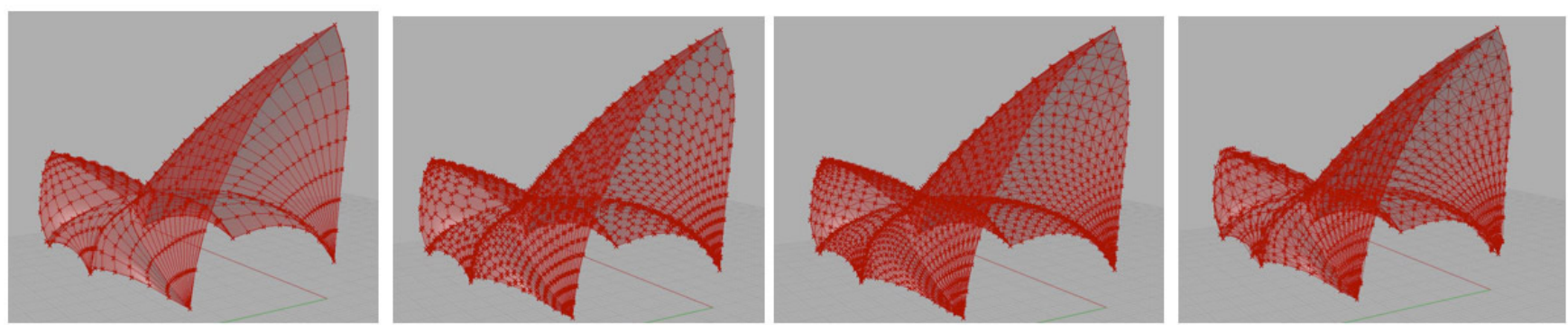

Figura 5. Aplicación directa del plugin LunchBox v3.o de Grasshopper, sobre la superficie de las cubiertas, considerando la geometría de la propuesta original de Jørn Utzon. Estudio de distintos patrones geométricos para un posible mallado de las superficies «libres» a través de barras.

- Mayor ligereza, lo que repercute no sólo en los esfuerzos que debe soportar la propia cubierta - cuya carga dominante es el peso propio- sino también en el tamaño de los pilares y cimentaciones bajo las mismas.

- Facilidad de prefabricación: unión de perfiles tubulares metálicos a través de soldadura en taller.

- Rapidez de ejecución: combinando la prefabricación de piezas de gran tamaño y uniones atornilladas en obra. Izado de módulos completos pre-montados a pie de obra.

- Drástica reducción de los medios auxiliares requeridos: debido a la mayor ligereza de las piezas a manipular en obra.

\subsection{Parametrización de posibles soluciones estructurales sobre las superficies de las cubiertas}

Una vez adoptada la decisión de plantear posibles soluciones en estructura metálica, se han aplicado técnicas de generación de geometrías tridimensionales con NURBS y de diseño paramétrico, con ayuda del programa informático Grasshopper sobre Rhinoceros, con el objetivo de plantear alternativas a la estructura de las cubiertas de la Ópera de Sídney desde una perspectiva contemporánea, tratando de respetar de forma lo más fiel posible la geometría de las cubiertas plasmada por Utzon en los planos presentados al concurso internacional de arquitectura convocado al efecto (geometría que no responde a ninguna forma conocida o expresable matemáticamente).

Aplicando varios algoritmos con la ayuda del programa informático Grasshopper, se han desarrollado diversas aproximaciones geométricas al problema de cómo disponer las barras de un entramado metálico adaptadas a las superficies «libres» planteadas por Utzon de modo que la estructura resultante sea lo más lógica y eficiente posible.

Se pretende realizar una aproximación sistemática en la que se puedan analizar en paralelo varias propuestas, parametrizadas, de modo que de una forma semi-automática se puedan extraer conclusiones acerca de la eficiencia de la disposición de dichas barras en la estructura.

De este modo, aplicando algoritmos pre-programados como LunchBox o Karamba se ha generado una familia de posibles formas, todas constituidas por barras de acero, adaptadas a la geometría original con la que Utzon ganó el concurso, para determinar de entre ellas cual es la disposición de barras más apropiada.

En primer lugar se han explorado las posibilidades de LunchBox v3.o, programado por Nathan Miller (ver Figura 5).
Empleando el algoritmo Karamba v1.O.2 se ha generado una geometría adicional, basada en una malla diagonal sobre la superficie libre propuesta por Utzon. En la Figura 6 se puede observar el resultado obtenido:

Una vez generadas las disposiciones de barras de las seis alternativas en estructura de acero (una propuesta inicial generada «manualmente» mediante corte de superficies así como las cinco adicionales anteriormente presentadas, generadas mediante algoritmos paramétricos), se realiza un análisis estructural de dichas propuestas en paralelo con ayuda del programa Autodesk Robot Structural Analysis v2012.

Las cargas consideradas han sido en los seis casos: el peso propio de los elementos estructurales de acero, una carga permanente de $1,0 \mathrm{kN} / \mathrm{m}^{2}$ correspondiente a los elementos de acabado de cubierta y una sobrecarga de mantenimiento de $0,4 \mathrm{kN} / \mathrm{m}^{2}$. En aras de mantener una cierta simplicidad a la hora de comparar los resultados obtenidos de los modelos de cálculo, no se ha tomado en consideración las cargas de viento y sismo, a pesar de que su acción podría suponer una influencia importante en el diseño final.

En la Figura 7 se presentan imágenes de los seis diferentes modelos de cálculo elaborados, cada uno de ellos con una disposición diferente de la malla sobre la superficie original planteada por Utzon:

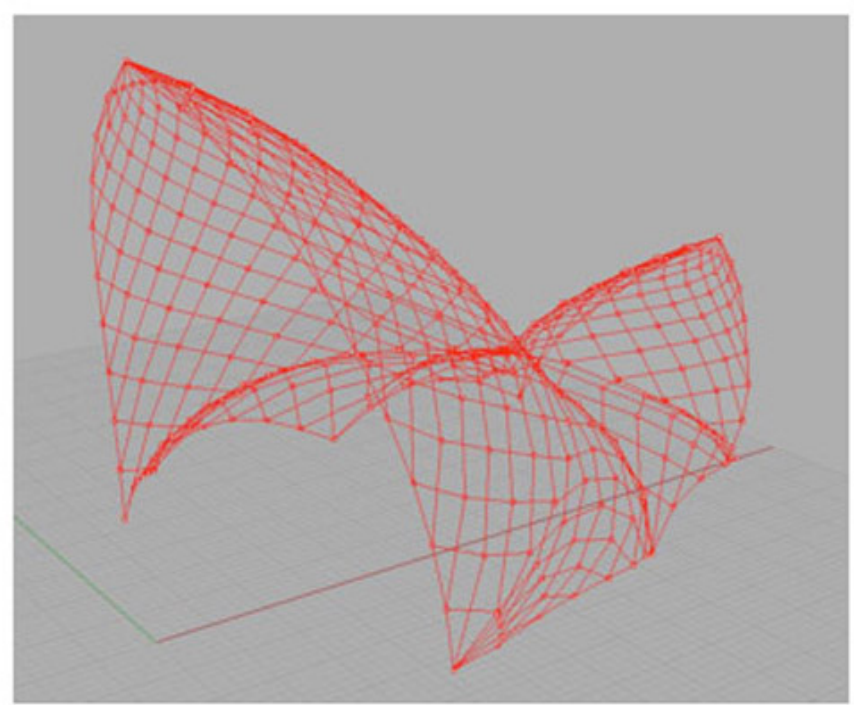

Figura 6. Aplicación directa del algoritmo Karamba de Grasshopper sobre la superficie de las cubiertas. 

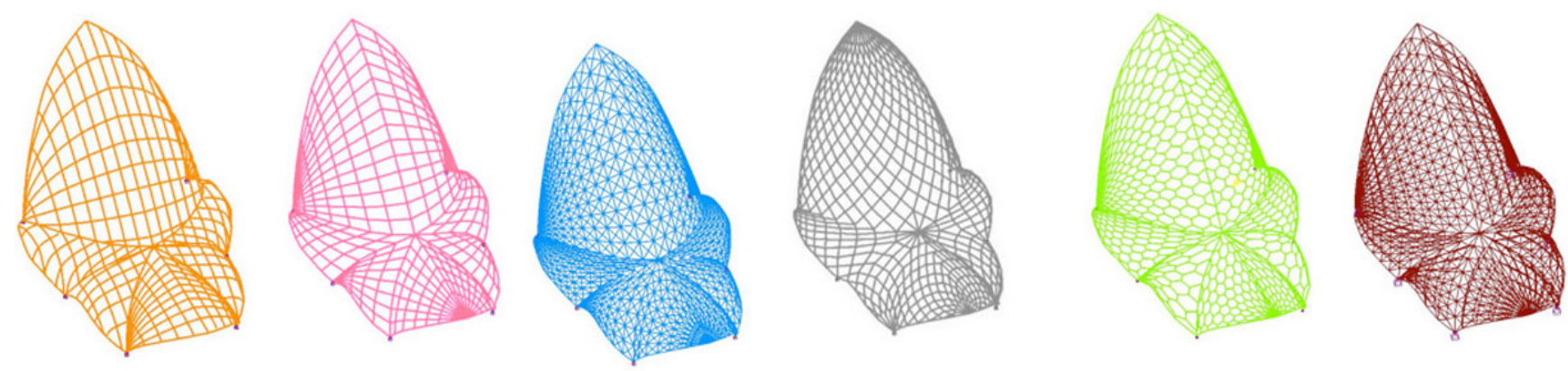

Figura 7. Vistas de los seis modelos de cálculo (de izquierda a derecha: A, B, C, D, E y F) elaborados en Autodesk Robot Structural Analysis v2012.

Se podría proceder pues a realizar un dimensionado en detalle de cada uno de los seis esquemas geométricos propuestos para, de su análisis comparado, determinar cuál es el más adecuado en términos de economía. En cualquier caso, se ha considerado que dicho análisis detallado excede los objetivos establecidos para la redacción del presente artículo, dado que la intención del autor es poner el acento en la exposición del proceso metodológico propuesto, especialmente el uso de las herramientas de generación formal, en detrimento de la determinación de forma precisa del tamaño de cada una de las barras de cada opción planteada.

De todos modos, de forma simplificada, se ha considerado un único tipo común de barras tubulares circulares (CHS) de acero S275-JR en todos los casos y, en base a estas premisas, se han analizado los seis modelos para de forma cualitativa poder clasificar los seis esquemas geométricos en función de su comportamiento estructural. La normativa empleada para realizar la comparación ha sido, de forma simplificada, el Código Técnico de la Edificación (CTE). Se han considerado uniones rígidas entre las barras y de forma simplificada se ha considerado un coeficiente de pandeo 1 para el dimensionado de las barras comprimidas.
En la Figura 8, como ejemplo, se presentan vistas de las geometrías deformadas obtenidas del cálculo de los seis modelos con un consumo similar de kg de acero en todos ellos.

A la vista de los resultados obtenidos se podría elegir la disposición geométrica considerada más adecuada en base a las premisas expuestas anteriormente.

Una vez realizada esta elección, podría afrontarse un nuevo análisis de sensibilidad sobre la densidad de la malla de barras. Para ello las herramientas de diseño paramétrico de nuevo se muestran de gran utilidad. Se podría realizar pues un nuevo análisis paramétrico para establecer, dentro de unos límites constructivos razonables, la densidad de malla más adecuada. Así, se generan una serie de opciones en función de la densidad de barras del modelo, simplemente alterando las variables de separación de las barras en cada una de las direcciones principales.

El objetivo sería de nuevo la minimización del peso de la estructura. El análisis se hace para un rango de separación de barras de entre uno y cuatro metros, por considerarse un abanico razonable para la correcta disposición del cerramiento de cubierta sobre ellas.
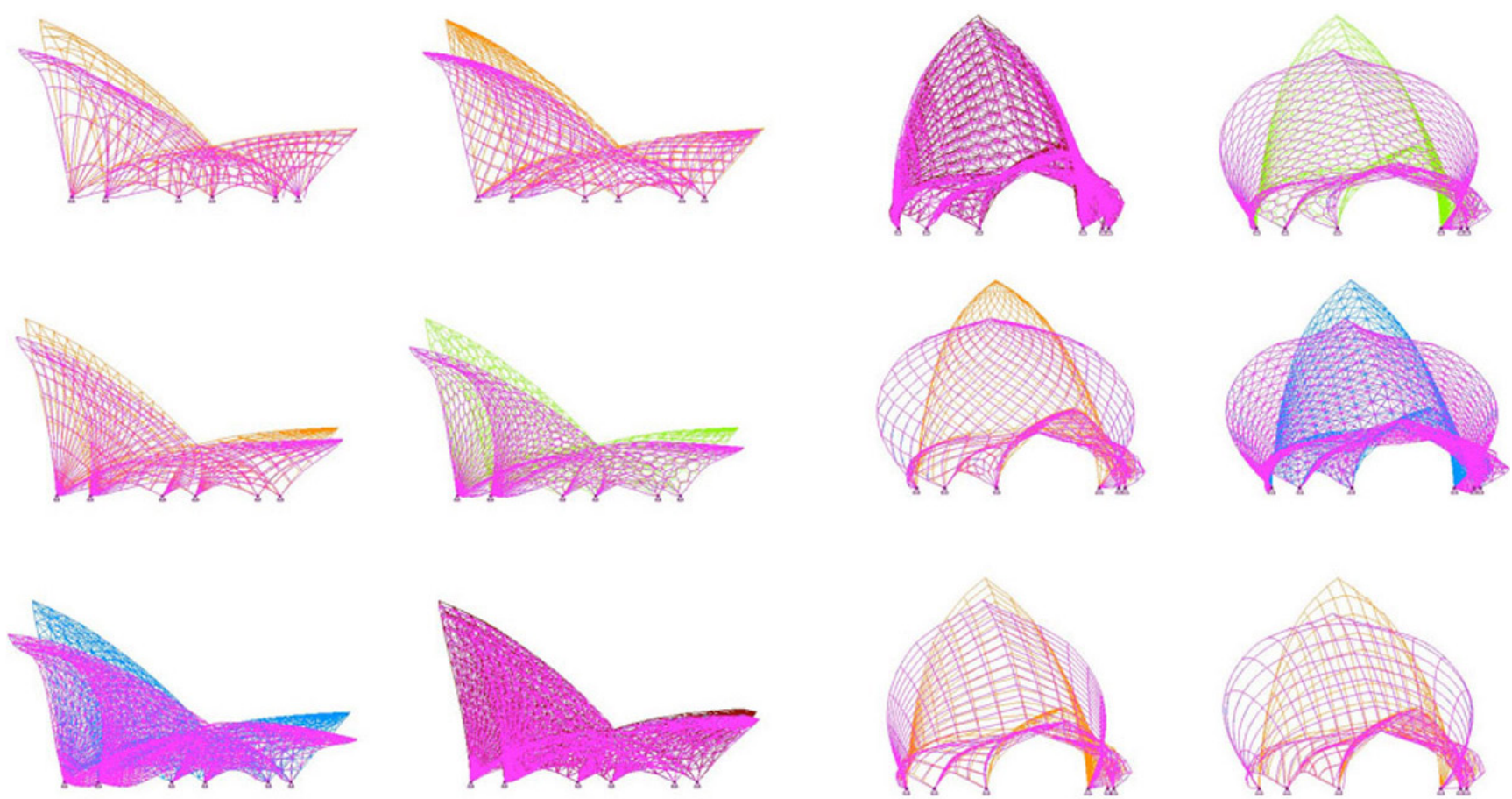

Figura 8. Geometría deformada (ELS) de los seis modelos de cálculo elaborados. De izquierda a derecha: fila 1: A D, F, E; fila 2: B, E, D, C; fila $3: \mathrm{C}, \mathrm{F}, \mathrm{B}, \mathrm{A}$. 


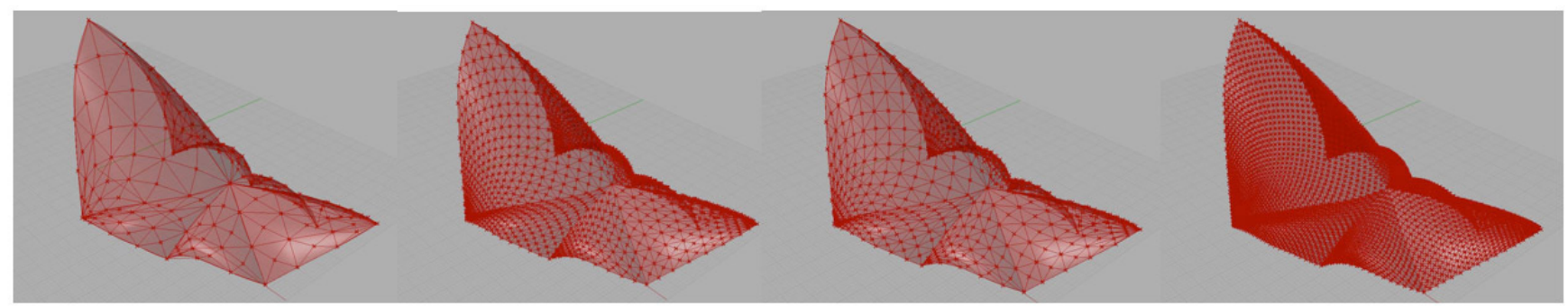

Figura 9. Aplicación directa del algoritmo LunchBox en Grasshopper. Estudio de distintos grados de densidad de malla (de izquierda a derecha: C1, C2, C3 y C4) para un posible mallado de las superficies «libres» a través de barras aplicado al patrón tipo C.

A continuación, en la Figura 9, se adjuntan unas imágenes de las nuevas formas generadas a partir de las premisas indicadas para la geometría tipo C.

Nuevamente se procedería a realizar un análisis estructural de las distintas propuestas con la ayuda del programa $A u-$ todesk Robot Structural Analysis v2012, con el objetivo de dimensionar las distintas opciones y poder realizar una comparación entre las mismas. Se adjuntan vistas de los tres modelos analizados (ver Figura 10).

Del análisis detallado de los resultados obtenidos se podría concluir cuál de entre las opciones estudiadas se considera la más adecuada por representar aquella que menor cantidad de estructura requiere.

Nótese que el hecho de tener un gran número de barras de dimensiones distintas no supone un inconveniente insalvable dado que, como se ha comentado, la estructura generada tridimensionalmente pasa directamente a las máquinas de corte, mediante sistemas CAD-CAM-CAE, las cuales afrontan de forma idéntica el corte de piezas de dimensiones repetitivas que de aquellas con gran variedad dimensional.

No obstante, es preciso recalcar la gran complejidad de montaje que representa una estructura de estas características,
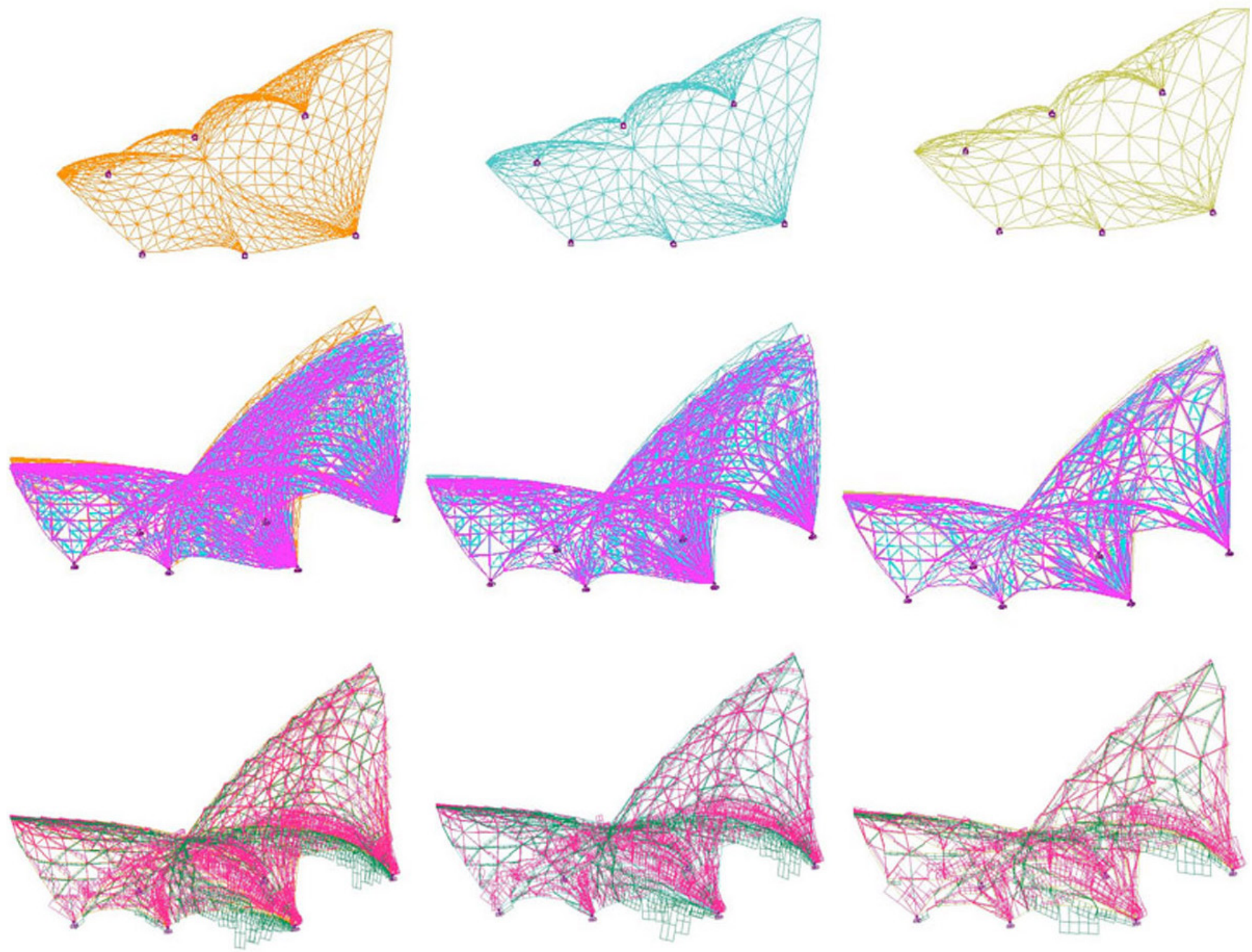

Figura 10. Modelos de cálculo elaborados. De izquierda a derecha; primera columna: patrón C2; segunda columna: patrón C3; tercera columna: patrón C1). De arriba abajo; primera fila: geometría inicial; segunda fila: geometría deformada (ELS); tercera fila: esfuerzos axiles en las barras (ELU). 
motivada fundamentalmente por la forma impuesta a la misma (en este caso la propuesta geométrica de Utzon para el concurso). Así mismo, existen nudos con gran densidad de barras que deberían analizarse en detalle para simplificar al máximo su diseño.

Lo que aquí se ha desarrollado pretende únicamente ser una aproximación a nivel de Anteproyecto a un problema de estas características. Sería necesario realizar un estudio más detallado para poder obtener conclusiones de mayor calado.

En cualquier caso, las herramientas de diseño paramétrico empleadas representan en este caso únicamente una ayuda para la generación rápida y automática de mallas de barras sobre una superficie predefinida. Esto es especialmente útil en estructuras en las que, como la objeto de estudio, por su complejidad formal resultan poco intuitivas. Como se ha expuesto, es posible hacer un análisis de alternativas de una forma relativamente sencilla que nos permita comparar la bondad de distintas disposiciones de barras sobre las superficies predefinidas, respetando los fuertes condicionantes formales existentes.

\section{CONCLUSIONES}

Las nuevas herramientas digitales de diseño paramétrico permiten, dentro del ámbito de las estructuras de edificación, la realización de análisis comparados de diseños estructurales de un modo manejable, posibilitando además su modificación de una forma relativamente sencilla. Esto posibilita que, incluso trabajando con formas o configuraciones espaciales complejas, se pueda llevar a cabo un proceso sistemático de análisis comparado de distintas propuestas estructurales tomando en cuenta los importantes condicionantes formales impuestos por el diseño en algunos casos.

Pero todo esto no debe hacernos perder de vista que, en realidad, no se trata más que de nuevas herramientas al servicio de la creatividad de los arquitectos e ingenieros y que son éstos en último término los responsables de hacer un uso sensato y responsable de las mismas. En palabras del arquitecto australiano Glenn Murcutt: «La mayoría de los arquitectos que construyen edificios extraños asegura que lo hacen porque ahora la tecnología lo hace posible. Eso me parece absurdo. Poder hacer una cosa no legitima hacerla» (18).

\section{REFERENCIAS}

(1) Rey-Rey, J., Fernández-Cabo, J.L. (2011). La desaparición de la barrera del análisis estructural en los proyectos arquitectónicos. Madrid: Congreso Hitos Estructurales de la Arquitectura e Ingeniería.

(2) Manterola, J. (1998). La estructura resistente en la arquitectura actual. Informes de la construcción, 50(456-457): 15-45, doi: http://dx.doi.org/10.3989/ic.1998.v50.i456-457.884.

(3) Martínez-Calzón, J. (2010). Treatment of the form in structural engineering. En Paulo J. S. Cruz (Ed.) Structures and Architecture. London: Taylor \& Francis group. doi: http://dx.doi.org/10.1201/b10428-4.

(4) Sasaki, M. (2004). Shape design of free curved surface shells. Número especial $a+u$, Architecture and Urbanism, 5(404): 36-37.

(5) Rey-Rey, J. (2013). La barrera del análisis estructural en el desarrollo de los proyectos arquitectónicos. El caso de la Ópera de Sídney (Tesis doctoral). Madrid: Escuela Técnica Superior de Arquitectura de Madrid-UPM.

(6) Utzon, J. (1971). Teatro de la ópera. Sídney. Informes de la Construcción, 24(231): 22-32, doi: http://dx.doi.org/10.3989/ ic.1971.v24.i231.3359.

(7) Drew, P. (1995). Sydney Opera House. Architecture in detail. London: Ed. Phaidon.

(8) Arup, O., et al. (1973). The Arup Journal. London: Ove Arup Partnership.

(9) Candela, F. (1985). En defensa del formalismo y otros escritos. Madrid: Ed. Xarait.

(10) Azagra, D., Bernabeu, A. (2012). La estructura de las formas libres. Informes de la construcción, 64(526): 133-142, doi: http://dx.doi.org/10.3989/ic.11.015.

(11) Schmacher, P. (2008). Parametricism as style. Parametricist manifesto. En XI Bienal de arquitectura de Venecia.

(12) Sakamoto, T., Ferré, A. (2009). From control to design. Parametric / Algorithmic architecture. Barcelona: Ed. Actar.

(13) Utzon, J. Sydney Opera House - New South Wales State Records, Digital gallery. http://gallery.records.nsw.gov.au/ index.php/galleries/sydney-opera-house/.

(14) Rey-Rey, J. (2013). The disappearance of the structural analysis barrier: the Sydney Opera House from a contemporary perspective. En ICSA 2013. Guimaraes.

(15) Williamson, S. (2003). The relative cost of unskilled labor in the United States, 1774-present. Economic History Services.

(16) Meyer, C., Sheer, M.H. (2005). Do concrete shells deserve another look? Concrete International, 27(10): 43-50.

(17) Cassinello, P., Schlaich, M., Torroja, J.A. (2010). Félix Candela. En memoria (1910-1997). Del cascarón de hormigón a las estructuras ligeras del s. XXI. Informes de la Construcción, 62(519): 5-26, doi: http://dx.doi.org/10.3989/ic.10.040.

(18) Zabalbeascoa, A. (2005). La arquitectura debe ser una respuesta, no una imposición. Diario El País. Edición de 18 de Junio de 2005, entrevista a Glenn Murcutt. http://elpais.com/diario/2005/o6/18/babelia/1119049574_850215.html. 